\title{
High power test of an injector linac for heavy ion cancer therapy facilities
}

\author{
Liang Lu, ${ }^{1, *}$ Toshiyuki Hattori, ${ }^{2}$ Huanyu Zhao, ${ }^{1}$ Katsunori Kawasaki, ${ }^{3}$ Liangting Sun, ${ }^{1}$ \\ Yuan $\mathrm{He}^{1}{ }^{1}$ and Hongwei Zhao ${ }^{1}$ \\ ${ }^{1}$ Linear accelerator group, Accelerator Division, Institute of Modern Physics, \\ 509 Nanchang Road, Lanzhou 730000, China \\ ${ }^{2}$ National Institute of Radiological Sciences, 4-9-1, Anagawa, Inage-ku, Chiba-shi 263-8555, Japan \\ ${ }^{3}$ Tokyo Institute of Technology, Nagatusda 4295, Yokohama 226-8502, Japan
}

(Received 5 February 2015; published 23 November 2015)

\begin{abstract}
A hybrid single cavity (HSC) linac, combined with radio frequency quadrupole and drift tube structure in a single interdigital-H cavity, operates with high rf power as a prototype injector for cancer therapy synchrotron. The HSC adopts a direct plasma injection scheme (DPIS) with a laser ion source. The input beam current of the HSC is designed to be $20 \mathrm{~mA} \mathrm{C}^{6+}$ ions. According to simulations, the HSC can accelerate a 6-mA ${ }^{6+}$ beam which meets the requirement of the particle number for cancer therapy $\left(10^{8 \sim 9}\right.$ ions/pulse). The HSC injector with DPIS makes the existing multiturn injection system and stripping system unnecessary; what is more, it can also bring down the size of the beam pipe in existing synchrotron magnets, which can reduce the whole cost of the synchrotron. Details of the field measurements of the HSC linac and results of the high power test are reported in this paper.
\end{abstract}

\section{INTRODUCTION}

Recently, heavy ion cancer therapy has proved to be a remarkably effective treatment. However, the fact is that the injection accelerators at existing facilities are large in size and expensive to run. The first generation of heavy ion cancer therapy facilities, i.e., the heavy ion medical accelerator in Chiba, has cured over five thousand patients [1]. Its linear accelerator part is over $30 \mathrm{~m}$ in length [2], whereas the linear injector length of a new generation of heavy ion cancer therapy facility at Gunma University, which began treatments in 2010, is around $6 \mathrm{~m} \mathrm{[3].} \mathrm{The} \mathrm{electron}$ cyclotron resonance source, used for existing heavy ion synchrotron facilities, can produce only hundreds of microampere $\mathrm{C}^{4+}$ ions, so the stripping system, which can make the beam emittance growth bigger, and a multiturn injection system are necessary to change the ion state from $\mathrm{C}^{4+}$ to $\mathrm{C}^{6+}$ and inject enough ions to the synchrotron. The injectors have to accelerate $\mathrm{C}^{4+}$ ions up to $6-8 \mathrm{MeV} / \mathrm{u}$ to obtain over $90 \%$ transformation rate of the $\mathrm{C}^{6+}$ charge state. In our research, we aimed to design a compact injection linac that was less than $2 \mathrm{~m}$ but had the ability to directly accelerate the high intensity $\mathrm{C}^{6+}$ ion beams by using the direct plasma injection scheme (DPIS). The DPIS could easily inject dozens of milliampere $\mathrm{C}^{6+}$ ions to the

\footnotetext{
*Corresponding author. luliang@impcas.ac.cn

Published by the American Physical Society under the terms of the Creative Commons Attribution 3.0 License. Further distribution of this work must maintain attribution to the author(s) and the published article's title, journal citation, and DOI.
}

linac $[4,5]$. The hybrid single cavity (HSC) injector, which is a coupled rf-IH cavity and are operating in FRANZ and MILAC [6,7], could accelerate $\mathrm{C}^{6+}$ ions from $25 \mathrm{keV} / \mathrm{u}$ up to $2.1 \mathrm{MeV} / \mathrm{u}$ in $1800 \mathrm{~mm}$ with less than $100 \mathrm{~kW}$ power. According to simulations, the HSC linac could accelerate a $5.98 \mathrm{~mA} \mathrm{C}^{6+}$ ion beam, which contains sufficient ions for cancer therapy [8]. Thus, it could be used directly as an injector without the stripping system and the multiturn injection system. The results of the low power tests, including the resonant frequency, electric (E) field distribution along the beam axis and the tuning effect matched well with the numerical calculations. The measured frequency of the cavity was $100.49 \mathrm{MHz}, 0.5 \%$ within the designed value. The measured best Q value was $95 \%$ of the simulated value. Last year, the HSC linac was transported to the Institute of Modern Physics (IMP) [9] of the Chinese Academy of Sciences, where a $250 \mathrm{~kW}$ rf power source and a high current laser ion source (LIS) [10] can be used for further HSC research. In the past few months, the high power $\mathrm{C}^{6+}$ beam was accelerated and analyzed. The accelerated energy and the accelerated beam current matched the calculations.

\section{DESIGN AND MANUFACTURE}

In addition to the main part of the HSC linac, which includes a 4-rod radio frequency quadrupole (RFQ) section and a 16-cell drift tube (DT) section, a ground base plate (GBP) is designed to combine the RFQ and DT sections. A $65 \mathrm{~mm}$ length of exit fringe field (EXITFF) and an alternating phase focusing (APF) structure are adopted for the beam focusing in the end of the RFQ rods and the DT section. To achieve the design goal of less than a 2-m long 
TABLE I. Final design parameters for the HSC linac.

\begin{tabular}{lcc}
\hline \hline & RFQ section & GBP + DT section \\
\hline Charge to mass ratio (q/A) & \multicolumn{2}{c}{$6 / 12\left(\mathrm{C}^{6+}\right)$} \\
Frequency (MHz) & \multicolumn{2}{c}{100} \\
Total length (mm) & \multicolumn{2}{c}{1800} \\
Power (kW) (MWS) & \multicolumn{2}{c}{93.98} \\
Q value (MWS) & \multicolumn{2}{c}{14577} \\
Undercut length (mm) & \multicolumn{2}{c}{150} \\
Kilpatrick factor & \multicolumn{2}{c}{1.8} \\
Number of cells & 41 & $1+16$ \\
Synchrotron phase (degree) & $-90 \rightarrow-30$ & $0,-60,-30,30,30$ \\
Input energy (keV/u) & 25 & 220 \\
Output energy (keV/u) & 220 & 2000 \\
Transmission & $65.4 \%$ & $45.7 \%$ \\
Input current (mA) & 20 & 13.1 \\
Output current (mA) & 13.1 & 5.98 \\
Cavity diameter (mm) & 280 & 650 \\
Cavity length (mm) & 679.58 & 1120.42 \\
\hline \hline
\end{tabular}

cavity, a substandard RFQ transmission ( $65.4 \%$ of the input of the HSC) is adopted. The DT transmission, which is calculated to be $45.7 \%$ of the RFQ output (DT input). Total accelerated beam current is $5.98 \mathrm{~mA}$. The reason for the large beam loss in the DT section can be due to the use of a 30 degree APF structure, which only covers half of the exit beam phase at the end of the RFQ. A tuner is installed to adjust the E field distribution of the HSC cavity in the RFQ side. The design parameters are shown in Table I.

The most distinctive feature of the HSC fabrication lies in the core accelerating parts which are shaped by a 5-axis numerical control machine. In particular, a center plate shown in Fig. 1(a), including all the DTs, DT-stems, both ridges of the RFQ side and the DT side, is shaped from a massive piece of copper shown in Fig. 1(b), for reducing the alignments and assembling errors. The accuracy of the core parts assembly is found to be less than $\pm 20 \mu \mathrm{m}$ using alignment tools. The tools are also shaped by the numerical control machine. A sandwich-type assembly method [11], shown in the left part of Fig. 2, is adopted. The right part of Fig. 2 shows an image of the interior of the HSC linac after the assemblies. All the parts were shaped in three weeks and the assemblies were easily finished in a few hours. With this fabrication method, it is easy to create a better cooling effect. According to our calculations, the HSC can operate in $\mathrm{cw}$ mode for $\mathrm{H}^{+}$beam acceleration because the power for $\mathrm{H}^{+}$beam acceleration is only one fourth of the $\mathrm{C}^{6+}$ beam acceleration. In fact, we had already operated a cw mode in 2007 for $\mathrm{He}^{+}$ions acceleration in a DT-type linac which was designed for $\mathrm{P}^{2+}$ acceleration and manufactured in the same method [12].

\section{LOW POWER TESTS}

The main purpose of the low power tests is to measure the rf properties and the E field distribution in the HSC cavity. During the first low power test in Japan, the measured frequency and $\mathrm{Q}$ value of the cavity are $100.49 \mathrm{MHz}$ and 13848, respectively, and the measured $\mathrm{Q}$ value is about $95 \%$ of the calculated value. Therefore, the assumed power consumption for the real operating conditions is $98.95 \mathrm{~kW}$ for the $\mathrm{C}^{6+}$ beam acceleration. In the second low power test at IMP after four years unserviced, the frequency remained as the first test, however, the measured Q value is $91 \%$ of the simulated value, which means the HSC linac needs $110 \mathrm{~kW}$ to accelerate the $\mathrm{C}^{6+}$ beams.

The E field strength of the HSC cavity can be obtained via means of the bead perturbation method [13]. In this research, the E fields in the DT and RFQ sections are measured separately, because the beam bore in the DT section was larger than the average aperture radius of the RFQ. In order to precisely measure E field distribution, several perturbation balls, i.e., $3.17,4.5$ and $8 \mathrm{~mm}$ diameter balls, are used to measure the E field strength [11]. As shown in Fig. 3, all the measured values of the E field in the DT section were normalized to 1 . From Fig. 3, it is obvious that the measured axial E field strengths of the DT section are in accordance with the simulated values given by Microwave Studio (MWS) [14].

The center of one pair of two adjacent RFQ rods, where one is supposed to measure the E field strength in the RFQ section, is outside the DT bore. Thus, a new position is adopted to measure the E field in the RFQ section using the $3.17 \mathrm{~mm}$ perturbation ball, $5 \mathrm{~mm}$ off the beam axis as shown in Fig. 4. Figure 5 shows the comparison of the measured and the simulated $\mathrm{E}$ field distribution [11]. It is clear that there is no peak in the gap between the RFQ rods and the GBP, as is expected (see the phase design of the first gap in Table I). It is also evident that most of the measured and the simulated peak strengths of the E field in the DT gaps are similar except the value of the fifth gap where an error of approximately 5\% is observed. The error is due to the fact that the distance of the synchronous phase change in the fifth gap is only 120 degree (30 degree to +30 degree), which results in the lengths of the fourth DT and the fifth gap being the shortest in the design, and hence the measurements are sensitive.

The E field strength in the RFQ section is purposely designed with a high value which is higher by $3 \%$ than the desired value [8]. Figure 6 shows two measurements of E field strength between two rods using the $3.17 \mathrm{~mm}$ perturbation ball [11]. From Fig. 6, it can be seen that the tuning effect is approximately $4 \%$, which implies that the ideal $\mathrm{E}$ field strength between the interrods can be adjusted by controlling the insertion length of the tuner. Both of the two measurements exhibit the four section structure (the radial matcher structure, the gentle buncher structure, the accelerator structure, and the EXITFF structure) clearly. The tuning effects of the E field strength in the DT section are shown in Fig. 7. It is easy to see that the tuned E field 


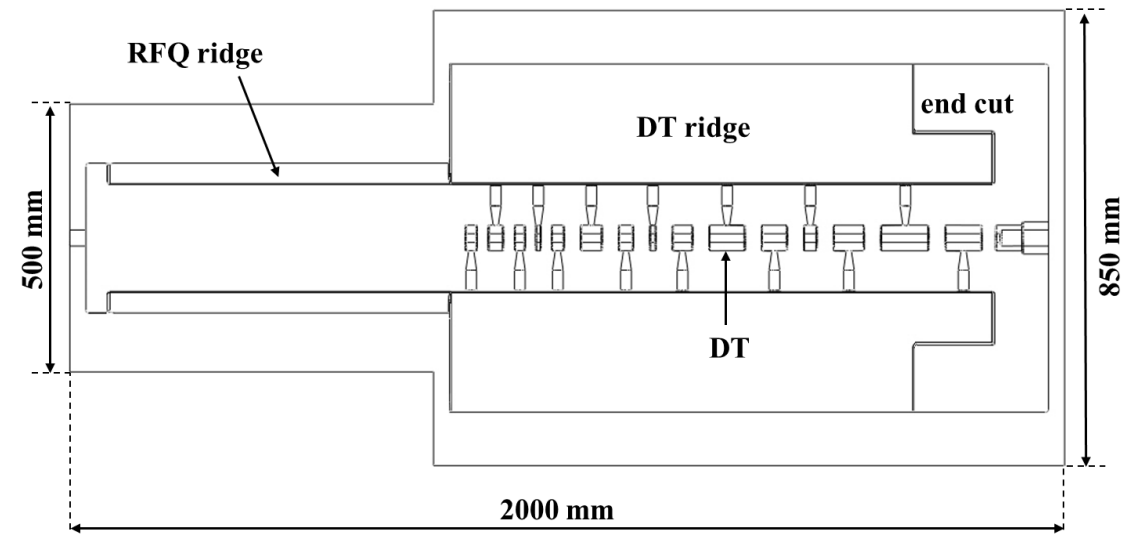

(a) an image of the shaped center plate which includes core parts of DT section.

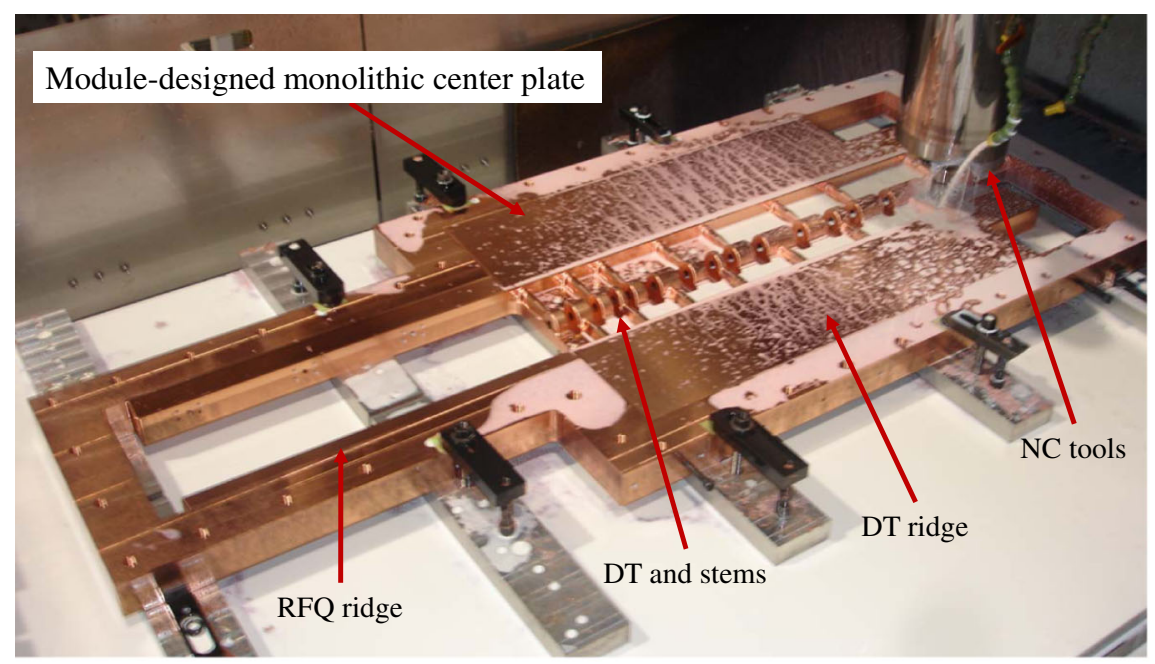

(b) An image of the center plate, which was being shaped by a 5-axis NC machine from a bulk

FIG. 1. Images of the designed and shaped center plate. (a) An image of the shaped center plate which includes core parts of DT section. (b) An image of the center plate, which was being shaped by a 5-axis NC machine from a bulk copper.
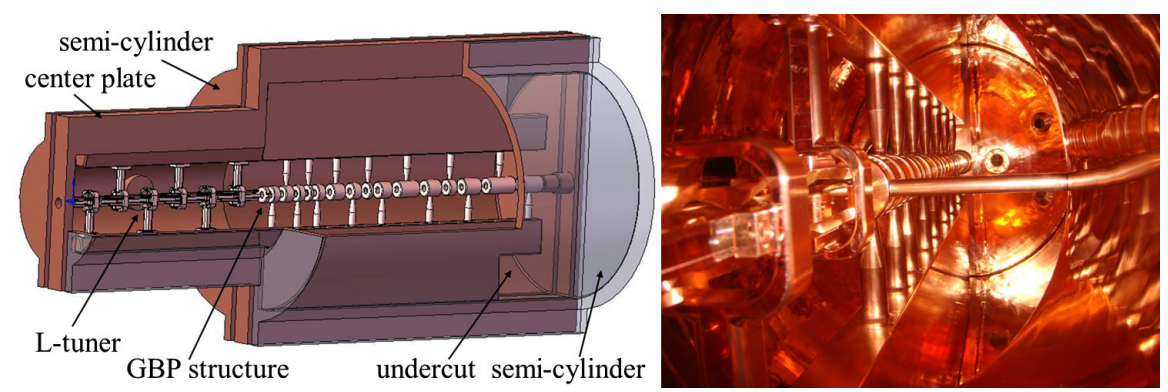

FIG. 2. Before and after assembly images of the HSC linac. The HSC linac was assembled by using a sandwich method (a center plate and two semicylinders).

strengths in the DT section are also 5\% higher than the simulated values, which meets the simulated results closely. The cavity frequency only increases by $0.01 \mathrm{MHz}$ when the maximum length tuner is inserted.
According to the perturbation measurements, the effective shunt impedance of the HSC is calculated as $111.2 \mathrm{M} \Omega / \mathrm{m}$, which is $91 \%$ of the simulated shunt impedance of $122.2 \mathrm{M} \Omega / \mathrm{m}$ calculated by 


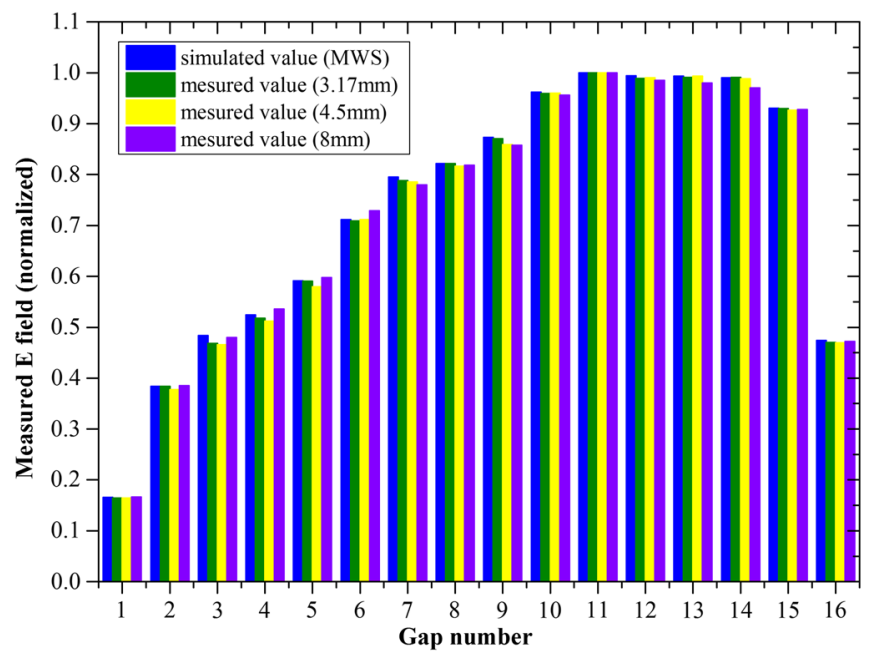

FIG. 3. Simulated and measured E field strengths along the beam axis in the DT section. The largest measured E field of the each measurement during the test was normalized to 1 .

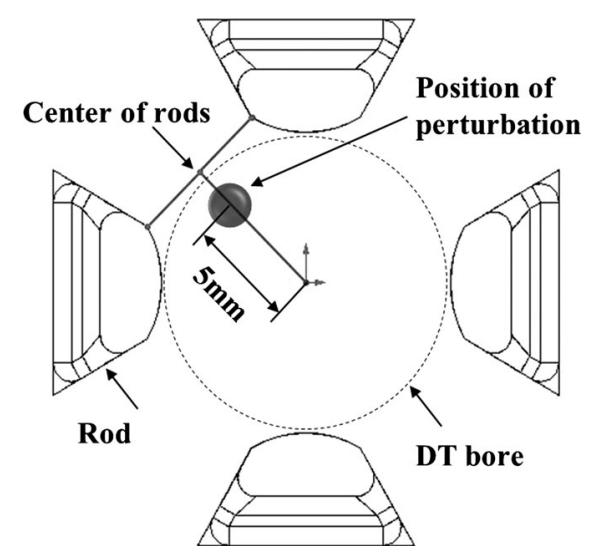

FIG. 4. An image of the new perturbation position for measuring the E field strength between interrods.

$$
\mathrm{R}_{\text {designed }}=\frac{\left[\left(E_{\text {out }}-E_{\text {in }}\right) /(\mathrm{A} / \mathrm{q}) / \cos 30^{\circ} / \mathrm{TTF}\right]^{2}}{P \times L},
$$

where the $P, L$ and TTF are the simulated power, cavity length and transit time factor, respectively. The shunt impedance of the HSC is a high value compared to RFQ's within the same beam velocity region, as shown in Fig. 8 [15].

According to the above measured results, the HSC cavity is successfully designed and assembled. The results also indicate that this convenient assembly method by using module design and numerical shaping can simplify the assembly process for accelerators with a high accuracy.

\section{HIGH POWER TEST AT IMP}

As shown in Fig. 9, the injection system consists of a LIS and a beam extraction system. The LIS can provide a

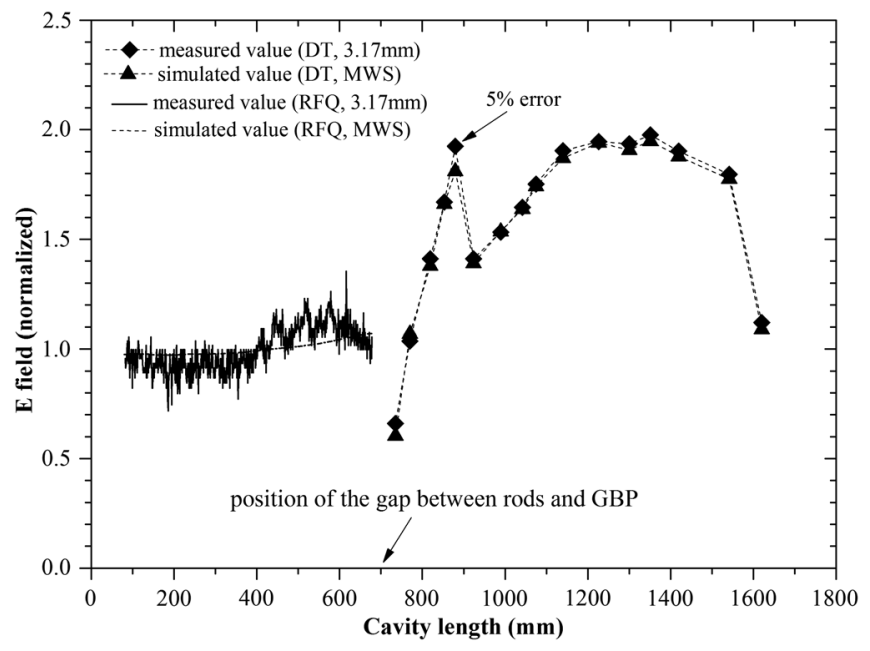

FIG. 5. A comparison of the measured and simulated E field strength along the axis of the new position of perturbation shown in Fig. 4. Both values of the measured and simulated $\mathrm{E}$ field in the RFQ shown in the figure were normalized to 1 . In this figure, the rhombic and triangular symbols represent the measured and simulated values of the E field in the DT gaps, respectively.

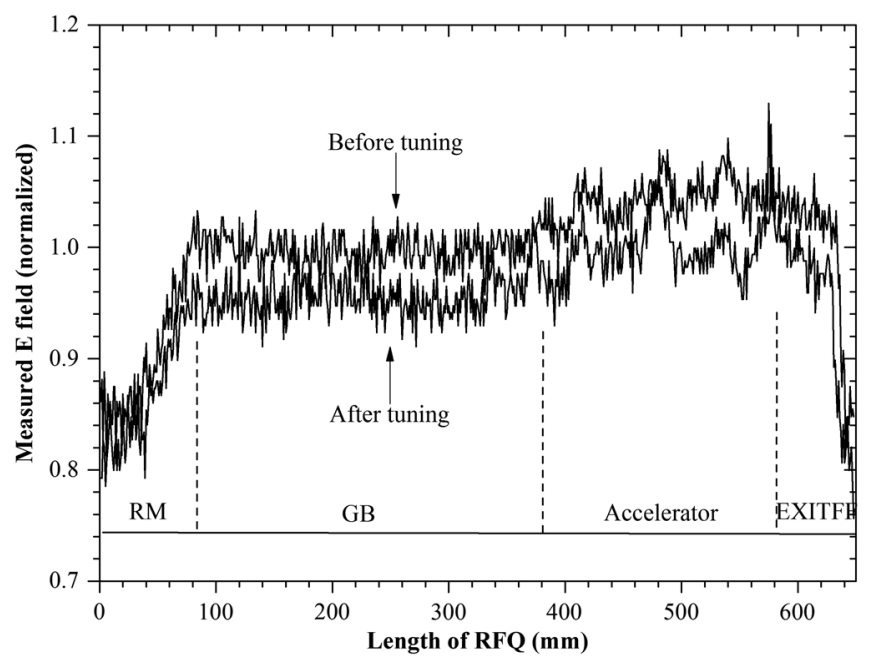

FIG. 6. Measurements of the E field strength between interrods before and after tuning.

maximum $20 \mathrm{~mA} \mathrm{C}^{6+}$ ion beam with a maximum of $60 \mathrm{kV}$ extraction voltage. The maximum laser energy is $3 \mathrm{~J}$, and the laser energy and power density are $1.9 \mathrm{~J}$ and $6.7 \times$ $10^{12} \mathrm{~W} / \mathrm{cm}^{2}$ at the focal point on the target, respectively. The target is a plate-type solid graphite target. High purity carbon products are very common and widely used for DPIS with a laser ion source for over 10 years. The manufacturing process can be found in the homepage of the Tokyo Tanso [28]. A voltage of $50 \mathrm{kV}$ is applied to the inner high voltage platform, which is connected with a plasma nozzle in order to inject the $\mathrm{C}^{6+}$ ions at the designed input energy of $25 \mathrm{keV} / \mathrm{u}$ into the grounded HSC linac. 


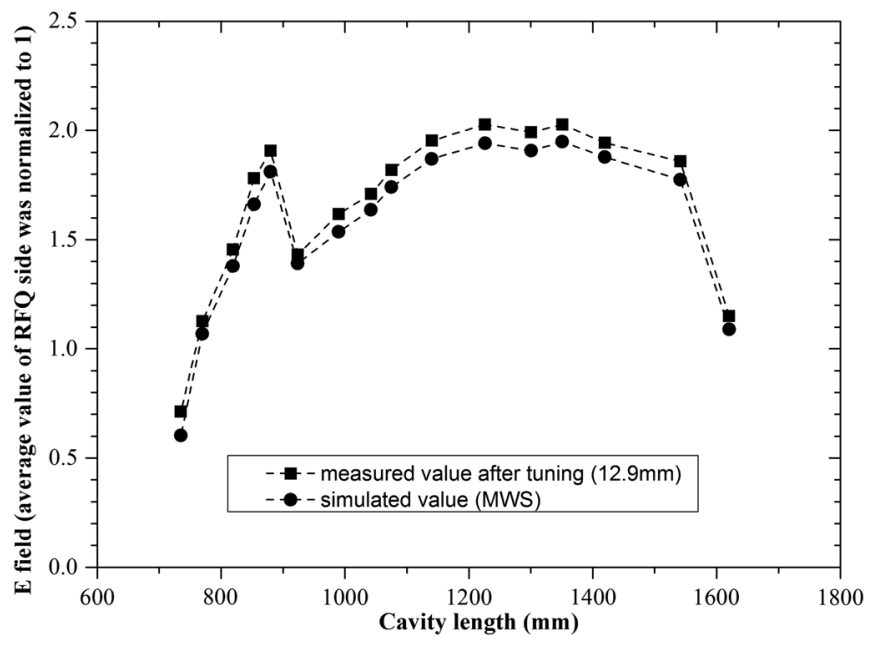

FIG. 7. Comparison of the measured and simulated E field strength in the DT section with and without the tuning.

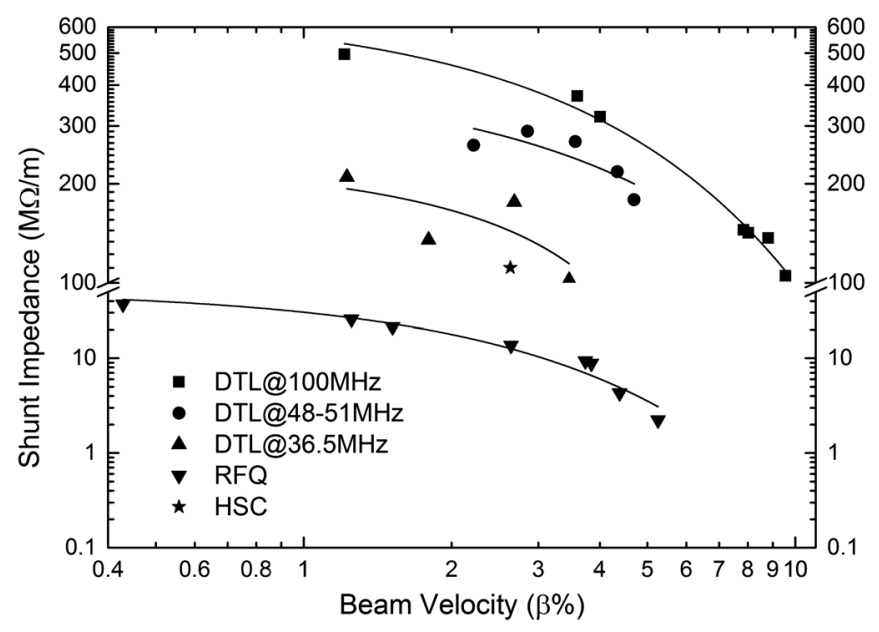

FIG. 8. Comparison of the shunt impedance of the HSC structured linac with that of other linac [16-27].

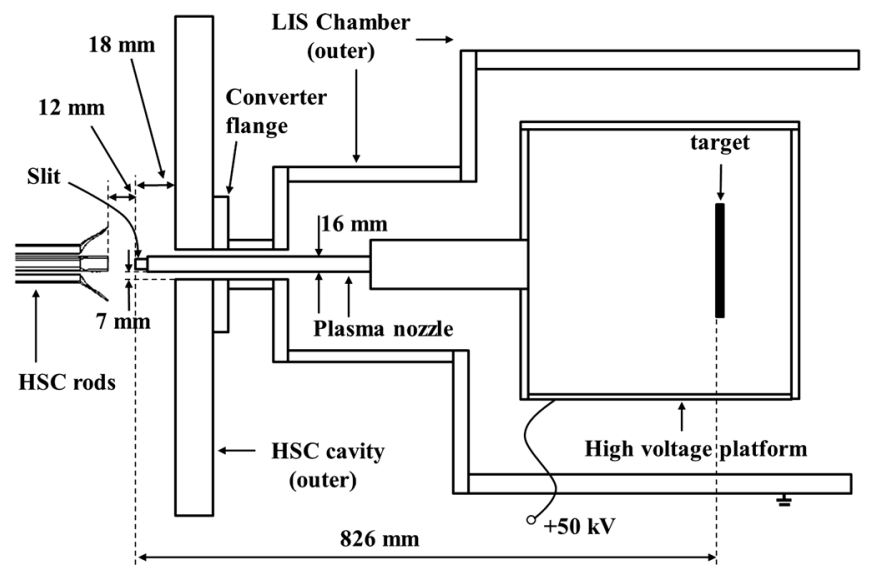

FIG. 9. The injection system for the HSC linac.

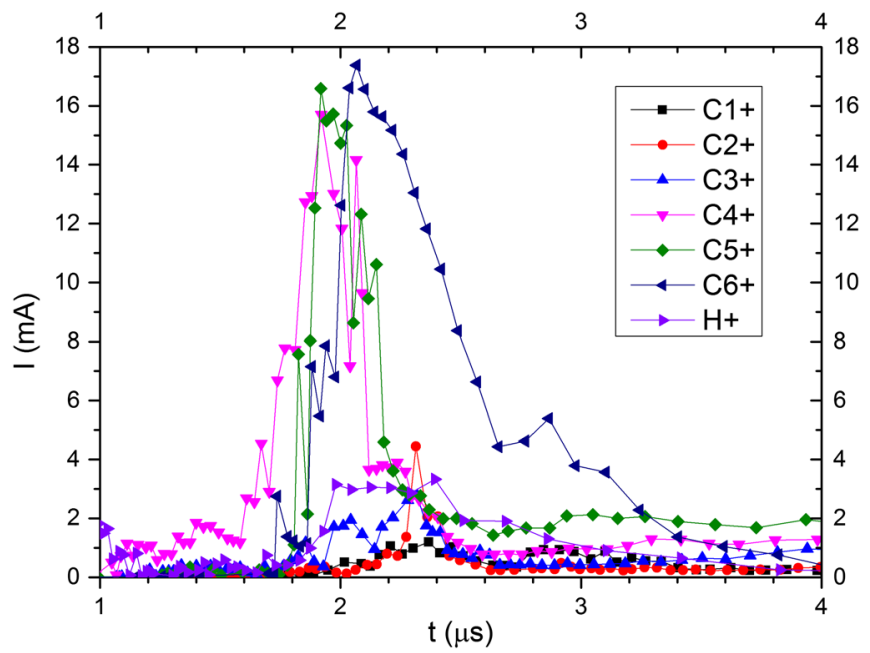

FIG. 10. The charge states from LIS, calculated position at the extraction slit shown in Fig. 9.

The generated plasma passes through the plasma nozzle with $50 \mathrm{kV}$ extractions and comes out from the nozzle tip. There is a length-adjustable slit installed on the tip, with a $7 \mathrm{~mm}$ inner diameter. The design injection point is the entrance of the rods, but in our injection system, there is a distance of $12 \mathrm{~mm}$ between the actual injection slit and the rods. $12 \mathrm{~mm}$ is a shortest distance for discharge limit. That means our system can inject $17.5 \mathrm{~mA} \mathrm{C}^{6+}$ ions to the RFQ rods. The charge states of the produced ions are shown in Fig. 10.

The commissioning of the cavity was performed with a duty factor of $1 \%$ until the incident power reached about $25 \mathrm{~kW}, 0.5 \%$ until the incident power reached about $80 \mathrm{~kW}$, $0.2 \%$ until the incident power reached about $105 \mathrm{~kW}$ and $0.1 \%$ until the incident power reached $110 \mathrm{~kW}$. Finally the incident $\mathrm{rf}$ power reached $165 \mathrm{~kW}$ and the resonated frequency changed $-27 \mathrm{kHz}$ compared with the initial state without rf power. In this high power test $110 \mathrm{~kW}$ was the operation point. The frequency change is very common when the cavity is aged and commissioned [22,29], and the cavity can tolerate the change. In our test system, we trigger the rf source with an external signal.

\section{BEAM ACCELERATION TESTS}

\section{A. Test system}

A beam acceleration system shown in Fig. 11 was built to measure the beam current. As shown in Fig. 12, trigger $\mathrm{A}$, trigger $\mathrm{B}$ and trigger $\mathrm{CD}$ from signal generator 1 (SG1) were used to trigger the laser, $\mathrm{Q}$ switch and the rf power, respectively. Trigger B was delayed by $680 \mu$ s to trigger A and $400 \mu$ s to trigger $\mathrm{CD}$, The time length of trigger $\mathrm{CD}$ was $1 \mathrm{~ms}$. In our operation, trigger B was considered as the starting point, and it was nearly the same as the diode trigger which was used to check the laser. The conditions of 


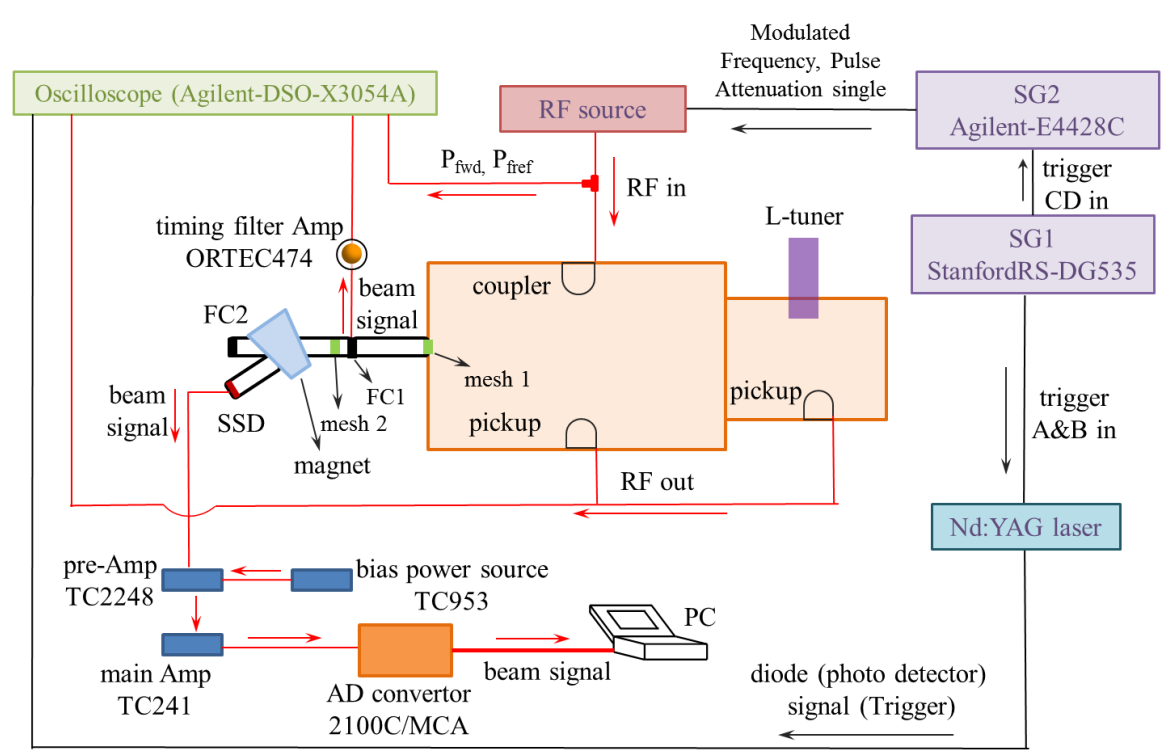

FIG. 11. Layout of the beam acceleration system.

the $\mathrm{rf}$ operation were $1 \mathrm{~ms}$ in pulse width and $0.1 \%$ in duty factor.

A Faraday cup (FC), $40 \mathrm{~mm}$ in diameter, located $2936 \mathrm{~mm}$ downstream from the target surface, was set to measure the total current after the HSC. This FC is an ordinary Faraday cup without the ability to measure the microbunching of the accelerated beams and to distinguish the peak times to the $\mu$ s level. Two solid state detectors (SSD), installed 45 degrees from the beam line behind the magnet, were also used to measure the beam signals. As shown in Fig. 11, a pre-Amp, a bias power source, a main Amp and an AD convertor were used to transfer the signal from the SSD to a computer. A power crate was used for the NIM electronics. Two locations, one at the exit of the HSC cavity (mesh 1) and another behind the FC (mesh 2), had meshes set to cut off the ions. The transmission efficiency of mesh 1 and mesh 2 are $50 \%$ and 5\%, respectively. The image of the whole high power acceleration system is shown in Fig. 13.

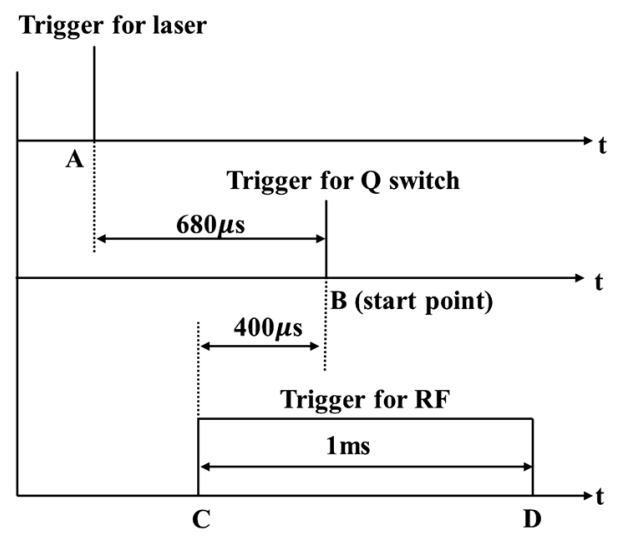

FIG. 12. The triggers from the SG1.

\section{B. Preliminary experiment}

The HSC is designed for $\mathrm{C}^{6+}$ ions accelerations, and the designed Kilpatrick factor is 1.8 , thus, the HSC can only accelerate $\mathrm{C}^{6+}$ ions and $\mathrm{H}^{+}$ions. The total accelerated current can be measured by the FC. In order to match the input energy to the input condition of the RFQ, it is necessary to measure beam with different extraction voltages. The compatibility condition of the input energy for the $\mathrm{HSC}$ linac is $25 \mathrm{keV} / \mathrm{u}$, and the highest accelerated $\mathrm{H}^{+}$ peak is measured at an extraction voltage of $25 \mathrm{kV}$ with a rf power of $27 \mathrm{~kW}$. As shown in Fig. 14, the peak appears at the extraction voltage of $20 \mathrm{kV}$, and disappears at the extraction voltage of $30 \mathrm{kV}$. That implies a voltage of $25 \mathrm{kV}$ is exactly the extraction voltage for $\mathrm{H}^{+}$beam injection. The $\mathrm{C}^{6+}$ beam commissioning is conducted with an extraction voltage of $50 \mathrm{kV}$ and $\mathrm{rf}$ power of $108 \mathrm{~kW}$. Figure 15 shows the measured beam pulse without extraction voltage and $\mathrm{rf}$ power, and a pulse of the accelerated $\mathrm{C}^{6+}$ ions with $50 \mathrm{kV}$ extraction and $110 \mathrm{~kW}$ rf power. The accelerated signal

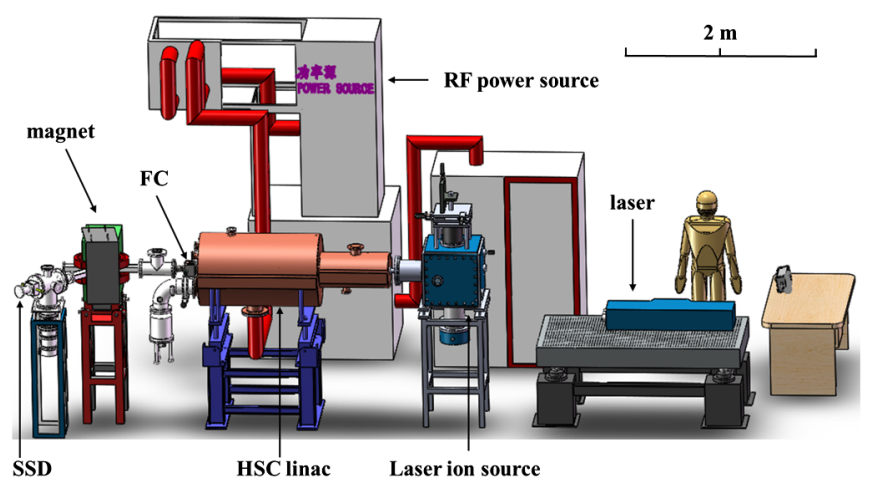

FIG. 13. The high power test system for $\mathrm{HSC} \mathrm{C}^{6+}$ ion accelerations. 


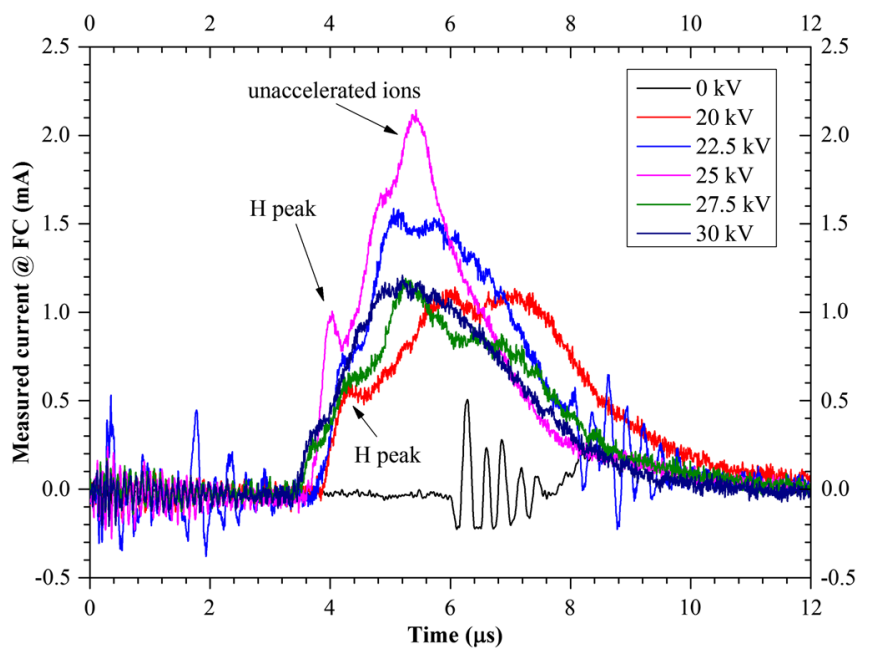

FIG. 14. Measured beam signals with respect to extraction voltages (plasma input position: $-12 \mathrm{~mm}$, rf power: $27 \mathrm{~kW}$ ).

was integrated over $100 \mathrm{~ns}$ by a timing filter. The peak of the accelerated $\mathrm{C}^{6+}$ ions reached $5 \mathrm{~mA}$, which agrees well with the simulations and transmission calculations. The transmission can be easily figured out as $5.03 \mathrm{~mA}$ by using the formula [30]

$$
T=\left(\frac{L_{1}}{L_{2}}\right)^{3} \times I \times T_{s},
$$

where $L_{1}$ is the existing length from the target surface to the injection slit, which is $826 \mathrm{~mm} ; L_{2}$ is the length from target surface to the RFQ rods, which is $838 \mathrm{~mm}$; I is the injection current of the $\mathrm{C}^{6+}$ ions at the position of $L_{1}$, which is $17.5 \mathrm{~mA}$ as shown in Fig. 10; and $T_{S}$ is the simulated transmission, which is $30 \%$ [8].

The calibration of energy channels for the SSDs is done by using a radioactive source: ${ }^{241} \mathrm{Am}$, which mainly emits

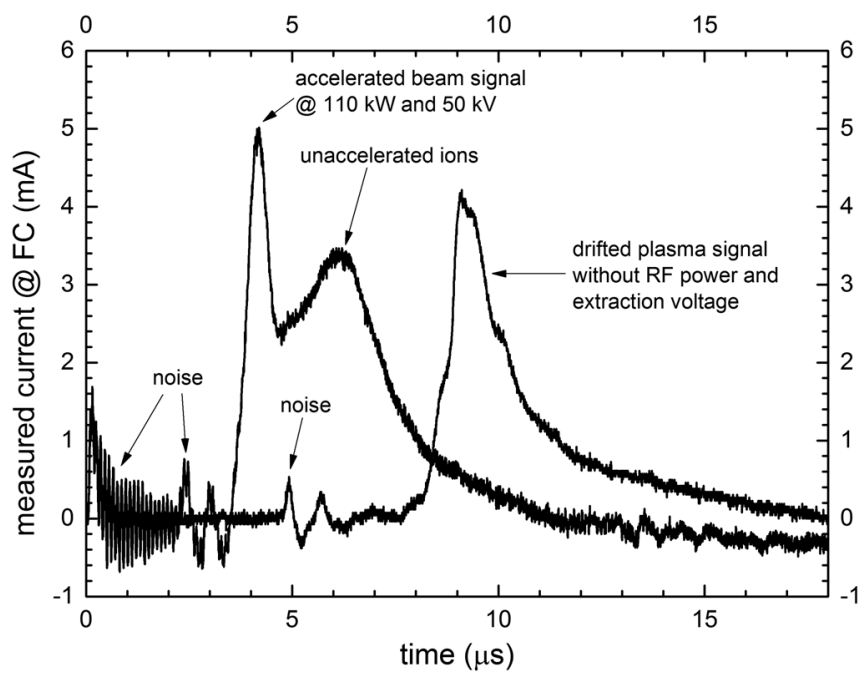

FIG. 15. Measured signals of the accelerated $\mathrm{C}^{6+}$ ion beam current and the unaccelerated ion beam current.

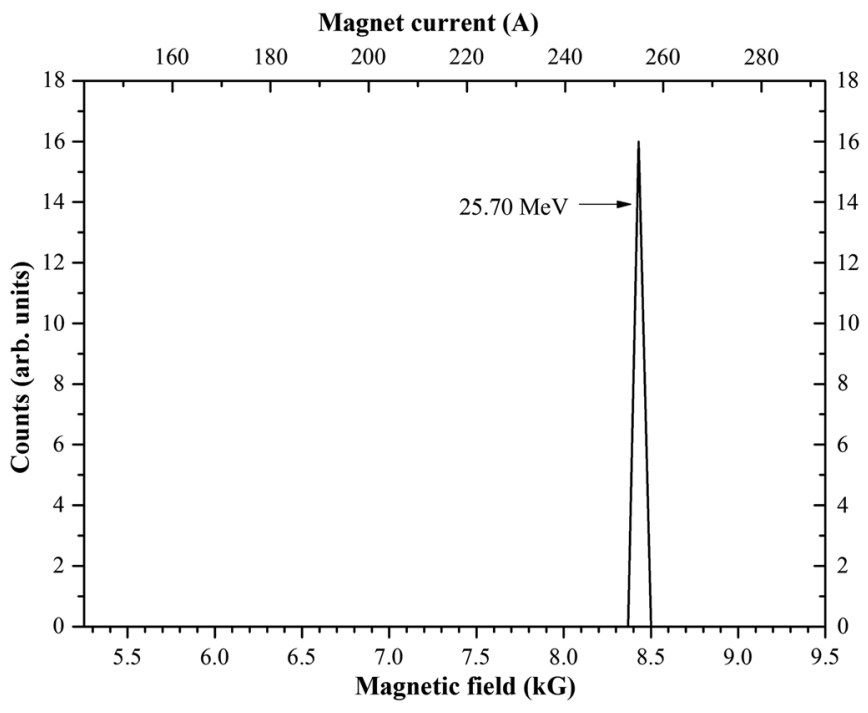

FIG. 16. Spectrum of energy distribution by ions moving through a bending magnet.

alpha particles with an energy of 5.486 MeV (85\%) [31]. In our preliminary experiments, the SSDs and the ${ }^{241} \mathrm{Am}$ are installed in a vacuum chamber for over $10 \mathrm{~min}$.

\section{Commissioning}

We used two SSD-type detectors to measure the beam energy. The SSDs were alternately installed on the beam axis downstream of the 45-degree-bending magnet. The bending magnet could provide a maximum 1 Tesla magnetic field with a maximum $300 \mathrm{~A}$ input current. The deflection radius of the magnet was $50 \mathrm{~cm}$. Using the SSDs, we checked the energy of the accelerated ions first by changing the input current of the magnet during operations with $110 \mathrm{~kW}$. As shown in Fig. 16, one spectrum was

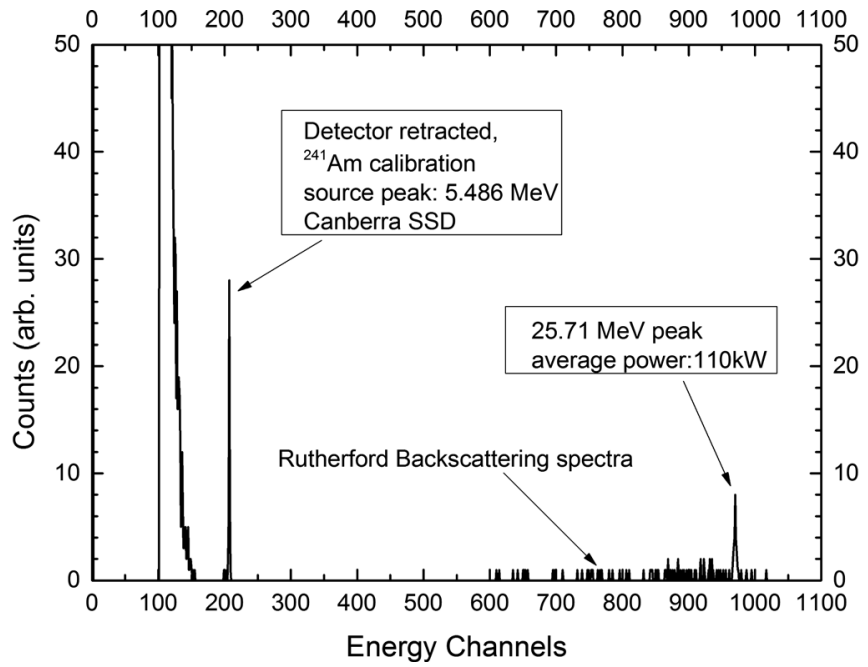

FIG. 17. Detected energy channels measured by the Canberra SSD. The $5.486 \mathrm{MeV}$ channel is the calibration source. The detected signal was calculated as $25.71 \mathrm{MeV}$. 
detected. The spectrum stands at 8.43 kilogauss which implies that the ions are at an energy of $25.7 \mathrm{MeV}$. This energy agrees well with the designed energy of $25 \mathrm{MeV}$ [8].

As shown in Fig. 17, the Canberra SSD confirmed the accelerated $\mathrm{C}^{6+}$ ion beams under the operations with $110 \mathrm{~kW}$, and showed the $\mathrm{C}^{6+}$ beam energy was $25.71 \mathrm{MeV}$ $(2.14 \mathrm{MeV} / \mathrm{u})$. The detected results of the beam energy agree well with the measured results shown in Fig. 16.

\section{CONCLUSIONS}

The HSC linac is proposed as a high intensity heavy ion injector had been proposed. We manufactured a 2-m long HSC linac as a prototype injector for cancer therapy synchrotron, and commissioned high power tests for the $\mathrm{C}^{6+}$ ion acceleration. The results of the preliminary tests and the commissioning tests agreed well with the designs and the calculations.

The results of the high $\mathrm{rf}$ power tests proved the HSC linac is reliable as an injector for heavy ion cancer therapy facilities. The results also encourage us to develop a new $\mathrm{HSC}$ as a real injector. The HSC injector would provide new developments for such a heavy ion cancer therapy facility.

\section{ACKNOWLEDGMENTS}

The authors would like to convey thanks to Dr. Zhouli Zhang and Dr. Yongming Li for fruitful discussions and comments on the platform construction. The authors are grateful to Dr. Liepeng Sun and Mr. Qianyu Jin and Mr. Junjie Zhang for their enthusiastic support in the operations of the rf power source and the laser machine.

[1] Y. Hirao, Nucl. Phys. A538, 541 (1992).

[2] E. Takada, Nucl. Phys. A834, 730c (2010).

[3] K. Noda et al., Nucl. Instrum. Methods Phys. Res., Sect. A 562, 1038 (2006).

[4] M. Okamura et al., High current RFQ using laser ion source, in Proceedings of XXII International Linear Accelerator Conference, Lübeck, Germany, August, 2004 (JACoW, Lübeck, Germany, 2004), pp. 315-317.

[5] H. Kashiwagi and T. Hattori, Rev. Sci. Instrum. 75, 1569 (2004).

[6] M. Heilmann et al., in Proceedings of the 4th International Particle Accelerator Conference, IPAC2013, Shanghai, China, 2013 (JACoW, Shanghai, China, 2013), THPWO017, pp. 3797-3799.

[7] V.O. Bomko et al., Coupling of parts accelerating structures $R F Q$ and $S T L$ in one resonator prestripping section the heavy ion linear accelerator MILAC, Problems of Atomic Science and Technology (2014), pp. 20-23; http://vant.kipt.kharkov.ua/ARTICLE/VANT_2014_3/ article_2014_3_20.pdf.

[8] L. Lu, T. Hattori, and Noriyosu Hayashizaki, Nucl. Instrum. Methods Phys. Res., Sect. A 688, 11 (2012).

[9] http://english.imp.cas.cn/.
[10] Z. Zhang, H. Zhao et al., in Proceedings of the Second International Particle Accelerator Conference, San Sebastian, Spain, 2011 (EPS-AG, Spain, 2011), pp. 128-130.

[11] L. Lu, T. Hattori et al., in Proceedings of the 5th International Particle Accelerator Conference, Dresden, Germany, June, 2014 (JACoW, Dresden, Germany, 2014), THPME025, pp. 3274-3276.

[12] L. Lu, T. Hattori, and N. Hayashizaki, Nucl. Instrum. Methods Phys. Res., Sect. A 622, 485 (2010).

[13] U. Ratzinger, Nucl. Instrum. Methods Phys. Res., Sect. A 464, 636 (2001).

[14] https://www.cst.com/Products/CSTMWS.

[15] T. Hattori, K. Sasa, M. Okamura, T. Ito, H. Tomizawa, T. Katayose, and M. Okada, Fusion Eng. Des. 32-33, 359 (1996).

[16] S. Arai, Y. Arakaki, Y. Hashimoto et al., in Proceedings of the International linear Accelerator Conference, Geneva, Switzerland, (JACoW, Geneva, Switzerland, 1996), WE203, pp. 575-579.

[17] T. Hattori, K. Sato, H. Suzuki et al., in Proceedings of 1986 International Conferencing on Linear Accelerators, Stanford, California, June, 1986 (Stanford University, Stanford, California, 1986), WE3-50, pp. 377-379.

[18] I. Takashi, H. Noriyosu, M. Shinjiro, S. Kimikazu, H. Schubert, E. Osvath, and H. Toshiyuki, Nucl. Instrum. Methods Phys. Res., Sect. B 161-163, 1164 (2000).

[19] K. Yamada, S. Arai, M. Fujimaki et al., in Proceedings of the 1st International linear Accelerator Conference, Kyoto, Japan, 2010 (JACoW, Kyoto, Japan, 2010), MOPD046, pp. 789-791.

[20] N. Ueda, T. Nakanishi, S. Arai et al., IEEE Trans. Nucl. Sci. 30, 4 (1983).

[21] M. Okamura, M. Okada, T. Ito, and T. Hattori, Nucl. Instrum. Methods Phys. Res., Sect. B 99, 694 (1995).

[22] P. N. Ostroumov, B. Mustapha, A. Barcikowski et al., Phys. Rev. ST Accel. Beams 15, 110101 (2012).

[23] D. Schrage, L. Young, B. Campbell et al., Nucl. Instrum. Methods Phys. Res., Sect. B 40-41, 949 (1989).

[24] Z. Zhouli, H. Yuan, S. Aimin et al., in Proceedings of the 5th International Particle Accelerator Conference, Dresden, Germany, June, 2014 (JACoW, Dresden, Germany, 2014), THPME027, pp. 3280-3282.

[25] K. Hasegawa, M. Mizumoto, and N. Ito, J. Nucl. Sci. Technol. 34, 622 (1997).

[26] J. M. Han, H. H. Lee, K. Y. Seo et al., in Proceedings of the 2nd Asian Particle Accelerator Conference, Beijing, 2001 (Institute of High Energy Physics, Beijing, 2001), pp. 159-161.

[27] S. C. Joshi, G. V. Kane, N. K. Sharma et al., in Proceedings of the 27th Linear Accelerator Conference, Geneva, Switzerland, Sept., 2014 (JACoW, Geneva, Switzerland, 2014), MOPP124, pp. 345-347.

[28] http://www.toyotanso.co.jp/Products/Mechanical_carbon/ manufacturing-process_en.html.

[29] T. Ishibashi, N. Hayashizaki, and T. Hattori, Phys. Rev. ST Accel. Beams 14, 060101 (2011).

[30] T. Kanesue, M. Okamura, K. Kondo, J. Tamura, H. Kashiwagi, and Z. Zhang, Rev. Sci. Instrum. 81, 02B723 (2010).

[31] C. Klinck, $\alpha$-decay of ${ }^{241}$ Am theory-A lecture course on radioactivity, University of Technology Kaiserslautern, 2010. 